June 2003

\title{
Care management for persistent pain: An introduction
}

Christine W. Hartmann

Thomas Jefferson University

Neil I. Goldfarb

Thomas Jefferson University

Susan S. Kim

Thomas Jefferson University

Bhaskar R. Nuthulaganti

Thomas Jefferson University

Raafat Seifeldin

Thomas Jefferson University

Follow this and additional works at: https://jdc.jefferson.edu/healthpolicyfaculty

Part of the Health Services Research Commons

Let us know how access to this document benefits you

\section{Recommended Citation}

Hartmann, Christine W.; Goldfarb, Neil I.; Kim, Susan S.; Nuthulaganti, Bhaskar R.; and Seifeldin, Raafat, "Care management for persistent pain: An introduction" (2003). College of Population Health Faculty Papers. Paper 9.

https://jdc.jefferson.edu/healthpolicyfaculty/9

This Article is brought to you for free and open access by the Jefferson Digital Commons. The Jefferson Digital Commons is a service of Thomas Jefferson University's Center for Teaching and Learning (CTL). The Commons is a showcase for Jefferson books and journals, peer-reviewed scholarly publications, unique historical collections from the University archives, and teaching tools. The Jefferson Digital Commons allows researchers and interested readers anywhere in the world to learn about and keep up to date with Jefferson scholarship. This article has been accepted for inclusion in College of Population Health Faculty Papers by an authorized administrator of the Jefferson Digital Commons. For more information, please contact: JeffersonDigitalCommons@jefferson.edu. 


\title{
Care Management for Persistent Pain: An Introduction
}

\author{
CHRISTINE W. HARTMANN, M.S.S., ${ }^{1}$ NEIL I. GOLDFARB, B.A., ${ }^{1}$ \\ SUSAN S. KIM, Pharm.D., ${ }^{2}$ BHASKAR R. NUTHULAGANTI, M.B.A., F.A.H.M., ${ }^{1}$ \\ and RAAFAT SEIFELDIN, Pharm.D., Ph.D. ${ }^{2}$
}

\begin{abstract}
Persistent pain is a frequently occurring condition with significant economic, clinical, and humanistic implications, for both individuals and society. Current literature, however, points to unresolved issues with regard to its identification, assessment, diagnosis, and treatment, and a number of suggestions have been made for improving the quality of care for pain sufferers. Because persistent pain shares many of the salient features of other chronic conditions such as diabetes and congestive heart failure, it is reasonable to believe that the adoption of a coordinated approach to care management could substantially improve the quality of care. Several strategies-including identification, appropriate referral, education, and planningcan and should be implemented to offer comprehensive, individualized treatment alternatives that are not currently available and that improve patient outcomes, including quality of life.
\end{abstract}

\section{INTRODUCTION}

$\mathbf{P}$ AIN IS GAINING RECOGNITION as a public health problem that has significant economic, clinical, and humanistic impact, on individuals as well as society. Pain has been estimated to cost the U.S. economy from $\$ 85$ to $\$ 90$ billion per year ${ }^{1}$ and is the second most common U.S. health problem resulting in work absenteeism. ${ }^{2}$ It has many different forms and names, including persistent pain and chronic non-malignant pain (CNMP); the differences between them are discussed specifically below. In studies, CNMP has been shown to affect anywhere from $2 \%$ to $40 \%$ of the general population, with the percentages being generally even higher for older adults in long-term care (LTC) facilities. ${ }^{3-5}$ Not only does CNMP have a significant rate of prevalence, it also often results in various detrimental effects on individ- uals' social, psychological, behavioral, and physical functioning. 6,7

Despite the multidimensional nature of the impact of pain, it has not received the same care management attention as some other high-impact conditions such as diabetes, congestive heart failure, and asthma. This can be explained partially by the fact that pain is a symptom that spans many disorders, rather than being a disorder itself. Nevertheless, persistent pain and CNMP do meet the usual criteria for identifying conditions that will most benefit from care management (eg, high prevalence, high impact, high cost, high variability in management and adherence, and high likelihood of success in improving outcomes given appropriate management). Care management involves an interdisciplinary coordination of services with a focus on maximizing individuals' functioning and independence. ${ }^{8}$ As persistent pain is often

\footnotetext{
${ }^{1}$ Office of Health Policy and Clinical Outcomes, Thomas Jefferson University, Philadelphia, Pennsylvania.

${ }^{2}$ Health Economics and Outcomes Research, Purdue Pharma, L.P., Stamford, Connecticut.
} 
under- or inappropriately treated, ${ }^{3,9}$ care management provides a method through which to identify a population at risk and to integrate individualized treatment across populations, conditions, and delivery systems. After a selected population is risk- or severity-stratified, appropriate care management interventions can then be targeted to individuals and groups in a cost-effective manner.

In 2000, the American Pain Society (APS) issued a statement supporting efforts by managed care organizations "to implement systematic methods of pain assessment and management to facilitate quality care and to obtain reasonable outcomes for pain management activities." 2 To achieve this outcome with regard to chronic pain, it recommended the incorporation of four principles: identification, appropriate referral, education of and assistance to primary care providers (PCPs), and the development of management plans similar to those in existence for chronic diseases. ${ }^{2}$ The APS suggested that the management plans should make use of guidelines and pathways, increasing the use of available resources and facilitating documentation of interventions' effectiveness. ${ }^{2}$ However, because few evidencebased guidelines currently exist with regard to CNMP, the APS suggested that managed care organizations devise their own, internal ones. ${ }^{2}$ Given the current situation in the United States with regard to the high incidence of pain and the lack of specific, targeted, and coordinated interventions, the effective implementation of selected care management strategies and principles could greatly improve the overall management of pain.

This paper presents a literature-based introduction to the impact of pain and issues surrounding implementation of care management activities specific to pain management. The intention is not to provide clinical guidelines and treatment recommendations but rather to offer an introduction for care management professionals, in order to encourage the development of appropriate pain management programs.

\section{PAIN}

A number of different terms are used to describe longer-lasting pain, though the inter- changeable nomenclature can cause confusion and lead to comparability problems in research. ${ }^{10}$ This paper, when possible, uses "persistent pain" - referring to non-malignant painto highlight the less negative connotation, the ongoing nature of sensations, and the frequent need for around-the-clock pain management associated with the term, although both "persistent" and "chronic" are used in the literature, without any consistent differentiation. ${ }^{11}$ According to the International Association for the Study of Pain, pain is "an unpleasant sensory and emotional experience associated with actual and potential tissue damage, or described in terms of such damage," and chronic pain is pain that "persists beyond the normal time of healing." 12 Chronic or persistent pain, therefore, is not associated only with specific physical injury or dysfunction but also has independent psychological and behavioral components. ${ }^{6,13}$ In addition, persistent pain has a different pathophysiologic etiology than acute pain, with that of persistent pain being less well understood. ${ }^{14}$ Some of the most common forms of persistent pain involve pain associated with recurrent headaches, low back pain, and arthritis. While it is clearly differentiated from acute pain because of its ongoing nature, in terms of duration persistent pain has been evaluated to be pain lasting for as little as 1 month or as much as 6 months, with some studies choosing to concentrate on lifetime prevalence. ${ }^{13,15}$

\section{CLINICAL IMPLICATIONS}

Numerous studies on CNMP have shown it to affect a significant portion of individuals, and it has been suggested that a prevalence estimate of $10 \%$ for the general population should be considered conservative. ${ }^{13}$ More individuals present in medical settings with non-malignant pain problems than with any other condition. ${ }^{16}$ Individuals with CNMP visit a healthcare practitioner 10.1 times in a 6-month period-as compared with 1.9 times for individuals without pain. ${ }^{17}$ Of those aged $20-64$ in the United States, 26 million have chronic back pain. ${ }^{18}$ Additionally, the prevalence of persistent pain in older adults has been estimated to be even higher than for the population at large. Adults over the age of 65 experience twice as 
many chronic pain conditions as those who are younger. ${ }^{19}$ A systematic review of studies to date revealed that $49-83 \%$ of residents of long term care (LTC) facilities experienced some form of pain. ${ }^{3}$ A study of all U.S. nursing home residents showed that $14.2 \%$ had persistent pain over two assessments and that of those who were in pain at the time of the first assessment, an average of $41.2 \%$ were in severe pain at follow-up 2-6 months later. ${ }^{20}$ In many studies a relationship has also been found between gender and the reporting of persistent pain. A large, multinational World Health Organization (WHO) study found that women across countries tended to have rates of persistent pain that were higher than those for men, although the reasons behind this trend remain elusive. ${ }^{15}$

With the exception of the case of individuals suffering from arthritis, however, persistent pain is not often associated with a demonstrable disease. ${ }^{16}$ This, along with an absence of adequate education on the part of healthcare consumers and providers and an absence of standards and guidelines for care, makes reliable identification and treatment of persistent pain difficult. ${ }^{9,21}$ In consequence, care management strategies used to identify and screen potential populations and then implement individualized treatment plans have great potential for addressing the needs of this underserved population. On a clinical level, it is imperative that both healthcare practitioners and consumers become more aware of the issues surrounding persistent pain. ${ }^{2}$

\section{ECONOMIC IMPLICATIONS}

The economic impact of persistent pain on the U.S. economy as a whole and on managed care organizations in particular is formidable. The economic impact of pain includes both direct and indirect costs-costs relating to utilization of goods and services designed to alleviate pain as well as those associated with inefficiency and impairment in on-the-job functioning. The total societal costs related to pain have been estimated to exceed $\$ 80$ billion per annum, and each year an estimated 4 billion workdays are lost.,22 These costs are shouldered predominantly by employers. Workers in pain not only take sick days but also are less likely to perform efficiently even when they are at work. ${ }^{23}$ Individuals suffering from persistent pain also have an increased usage of healthcare provider services, including emergency department visits and inpatient stays. ${ }^{16}$ Workers' $^{\prime}$ compensation systems on both the state and the private level are affected by the high rates of disability associated with persistent pain. ${ }^{2}$

In total, the annual costs associated with persistent pain exceed even those related to many major, financially burdensome, conditions. ${ }^{24}$ Specifically, taken together, three persistent pain conditions (back and neck pain, facial pain, and headache) have a total annual ageadjusted mean cost of $\$ 13,303$, as compared with costs of $\$ 7,626, \$ 5,649, \$ 6,554, \$ 10,246$, and $\$ 13,139$ for heart disease, hypertension, respiratory disease, HIV infection, and stroke, respectively. ${ }^{24}$ The percent increase in healthcare cost for persons with these three persistent pain conditions, compared with costs for individuals without the conditions, varies from $136 \%$ to $187 \% .{ }^{24}$ While cost should not be the only factor in decisions related to health care, the delivery of cost-effective services is crucial to the improvement of health outcomes in the United States, where determinations about care are frequently closely linked to their economic impact. As chronic conditions account for a large percentage of healthcare costs in general, focusing on more effective and accurate assessment and treatment of persistent pain is one method of reducing economic strain for individuals as well as society.

\section{HUMANISTIC IMPLICATIONS}

In addition to the economic burden pain places on individuals and society, with it also comes an increased risk of a reduction in quality of life. Pain leads to a lowering of mood and the ability to function in daily roles. ${ }^{25}$ Persistent pain can cause an individual to withdraw from previously pleasurable and satisfying activities as well as impede her/his capacity to fulfill personal, familial, and societal expectations. The previously cited multinational WHO study found that persons with persistent pain were likely to have missed 3 or more days in the previous month from their usual activities. ${ }^{15}$ A significant association between persis- 
tent pain and psychological disorders such as depression and anxiety was also found: "After adjusting for center [research site], age, sex, and physician-rated severity of physical disease, the odds of having a psychological disorder meeting diagnostic criteria among persons with persistent pain showed a 4-fold increase over those not affected by persistent pain."15 This relationship between pain and psychological dysfunction has also been documented for older adults living in LTC facilities. ${ }^{4}$ Older individuals are already more likely to suffer from conditions that often lead to persistent pain, such as arthritis, bone and joint disorders, and back problems, making this population especially vulnerable to the prospect of decreased quality of life. In addition, having persistent pain is associated with higher risks of depression and other psychological disorders, which negatively impact quality of life and also have been shown to be associated with growth in overall healthcare expenditures.

\section{NEED FOR IMPROVING THE MANAGEMENT OF PAIN}

Given the prevalence of pain in the general population, the high costs associated with pain treatment and disability resulting from pain, and the serious impact persistent pain has on quality of life, the lack of concentrated attention devoted to the issue and the high incidence of undertreatment is surprising. In late 2000, the U.S. Congress declared this to be the Decade of Pain Control and Research, and pain has been acknowledged in many circles to be the fifth, and equally important, vital sign; yet increases in knowledge about pain and the development of tools with which to assess and treat pain have not resulted in corresponding improvements in the delivery of adequate care to individuals. Pain remains undertreated, and four out of 10 individuals suffering from moderate to severe chronic pain are not provided with adequate pain relief. ${ }^{18}$ With regard to older individuals, a study which documented that $26 \%$ of LTC residents suffer from daily pain represents a fairly typical finding. ${ }^{4}$ In this study, of the residents who had CNMP, 25\% did not receive any analgesics at all. These data point to serious shortcomings in the present system of managing pain. Inadequate knowledge and misunderstandings about the nature of persistent pain, limited availability of assessment strategies and guidelines, deficiencies in the areas of training and skills, too little use of pain medications and pain relieving therapies, and a lack of interdisciplinary cooperation, on the part of both consumers of health care and the medical community, are all areas that need to be addressed.

\section{CASE FOR CARE MANAGEMENT SPECIFIC TO PAIN}

Care management emphasizes patient and provider education and the coordination of services and interventions, in order for treatment to take place appropriately, across the spectrum of care, without gaps in provision. Improved identification, screening, and provision of services could help reduce the problems and suffering associated with persistent pain, provide a more accurate picture of the size and characteristics of the population burdened by the condition, and reduce direct medical costs and the economic strain of lost productivity. Implementation of care management principles also could reduce costs through earlier detection and treatment, thereby reducing the need for long-term and increasingly expensive services, facilitating the targeting of care on an individualized basis, and reducing overlap of services.

With the enormous clinical, financial, and humanistic impact of persistent pain, its continued undertreatment and underdiagnosis pose significant problems. Strategies should be developed and implemented in order to address these concerns, and care management principles offer tools with which the current situation could be improved. The implementation of care management principles in relation to persistent pain would mirror the suggestions made by the APS. By including care management in an integrated system of healthcare delivery, implications of prior research and suggestions for improvement of care could be disseminated and serve to heighten the consumer and provider awareness of persistent 
pain as a condition. Care management would also focus attention on increased interdisciplinary coordination and cooperation to identify potential populations, screen and assess them, design individualized care management plans, implement them, and then monitor and evaluate the effectiveness of the outcomes. For example, indicators developed by an expert panel could be used to screen and identify patients presenting with persistent pain, making initial evaluations more efficient. Once patients were identified, a broad array of presently available interventions could be used to enhance patient care, producing not only better financial and clinical results, but also higher patient satisfaction.

Currently, there is some evidence that the quality of life for persistent pain sufferers would be improved through treatment provided at as early a point in the progression of the condition as possible. ${ }^{25,26}$ However, every step in the process of care for persistent pain could benefit from improvement. Implementation of care management principles can offer individuals with persistent pain, as well as providers, access to resources that are not widely utilized at present, but efficiently and effectively identifying and stratifying those in need remains a challenge to be overcome. In addition, quality improvement initiatives, outcomes measurement, and cost containment have yet to be well integrated in the model of care. Only with a systematic, well-coordinated, and practicable vision will the suffering of individuals with persistent pain be alleviated. Care management principles, consistently applied, provide the foundation for improving outcomes for individuals living with this prevalent, economically burdensome, and potentially debilitating condition.

\section{CURRENT MANAGEMENT}

Although the adoption of an interdisciplinary mode of care for persistent pain is being increasingly stressed, the relief of persistent pain through the use of analgesics and other medications remains an important component of any set of intervention principles, and a variety of pharmacological interventions ex- ist. ${ }^{27,28}$ Opioids continue to be a mainstay in terms of treating moderate to severe persistent pain, but other medications, not indicated in cases of acute pain, have also been found to be effective, including antidepressants, antiepileptics, and local anesthetics. ${ }^{14,29}$ Acute pain and persistent pain differ substantially and require different approaches, making management issues inherently more complex in the case of persistent pain. Long-term, around-theclock control of pain should be the focus of treatment, with the use of as needed medications limited to acute episodes. ${ }^{29}$ Yet despite the availability of a large variety of medications, they are often underused, in part because of patients' and providers' concerns about addiction, tolerance, regulations about and diversion of controlled substances, and side effects. ${ }^{28,30-32}$ At least partial resolution of these issues could be achieved through care management's standard of individualized treatment plans and coordination of care. In this regard, the continued education of PCPs-often the first to see or the ones to monitor patients in pain - on issues related to the symptoms and treatment options of persistent pain can help individuals find relief. ${ }^{27}$ The APS advocates "timely and effective assessment and treatment of pain" by PCPs, and for referrals to specialists when needed. ${ }^{2}$

While medications, when used appropriately, can be very effective in providing relief from pain, the treatment of persistent pain should not be limited to the use of pharmacologic interventions alone. At present, attention is being devoted to developing and implementing pain management standards and guidelines, but there are no widely used evidence-based guidelines dealing with persistent pain. ${ }^{2}$ Numerous instruments exist for assessing pain,, 33 but the more widespread and fundamental issue of systematically identifying and assessing potential populations remains relatively unaddressed.

The effectiveness of multidisciplinary pain treatment centers has been documented, and they are highly regarded in terms of the treatment of persistent pain. ${ }^{34,35}$ In these settings, treatment usually involves a number of coordinated services, often cognitive-behaviorally based, including education, stress manage- 
ment, relaxation, and biofeedback, in addition to medication management. While not all studies report clinically significant differences between treatment and control groups, ${ }^{34}$ several studies have shown reductions in patients' pain severity, mood, and physical impairment after the completion of a multidisciplinary pain management program. ${ }^{36-39}$

For example, results from one randomized controlled trial examining in- and outpatient treatment programs for persistent pain showed that patients who attended one of the two programs had a significant reduction in average pain intensity compared with those in the control group. ${ }^{35}$ Treatment for inpatients included education, behavioral pain management, cognitive restructuring, counseling, medication management (if needed), and positive reinforcement; outpatients attended an educational program including many of the components listed above; and patients in the control group were given standard medical treatments and assessments for a year. ${ }^{40}$ Although the study sample size was relatively small and the design suffered from a number of limitations, it provided evidence that multidisciplinary programs can be effective in improving overall functioning for persistent pain sufferers and that more intensive programs achieve stronger results. Another study of a multidisciplinary pain program conducted in a military hospital setting found a significant drop in the number of clinic visits for participants of the program after the first 3 months of treatment, though this study had no control group. ${ }^{16}$

Despite these encouraging findings, multidisciplinary programs remain out of the reach of many patients because the treatment is too expensive or programs are not covered by their health insurance. ${ }^{34}$ Additionally, such programs are unavailable to a significant proportion of persistent pain sufferers because their condition remains undiagnosed. Underdiagnosis is a pressing concern in the arena of persistent pain, and continued development and use of methods for screening populations and identifying potential sufferers are imperative. However, even for those who have been diagnosed, inadequacies may still occur, as services relying solely on evidence-based approaches may not address the multifaceted nature of the con- dition or the comorbidities associated with it. A diagnosis of persistent pain carries with it at a minimum a higher risk for conditions such as depression, anxiety, and sleep disorder, and in the worst cases, it may lead to serious disabilities. ${ }^{4,25}$ Care for persistent pain needs to revolve not only around population-based issues related to prevalence and evidence-based interventions, but also needs to reflect the broad nature of the condition and the necessity for individualized care management.

\section{CONCLUSIONS}

The idea of developing care management activities specifically around a symptom such as pain, rather than a disease, is controversial. However, the literature demonstrates that the prevalence and impact of pain exceed those of many diseases traditionally addressed through care management activities. Care management can step forward to fulfill an important role in relation to and address issues raised by the current state of persistent pain identification and treatment. While certain barriers to effective pain control exist, they are not insurmountable, and an examination of them can lead to a better understanding of the obstacles care management needs to address. Barriers can be divided into those relating to either attitude or aptitude: attitudinal ones include lack of interest, open-mindedness, and priority, while those related to aptitude pertain to lack of knowledge and skill. ${ }^{18}$ By definition, care management involves education, prioritizing individual needs and services, increasing the application of individualized treatment, and improving outcomes. It, therefore, provides a useful outline of methods to overcome difficulties and improve the current state of persistent pain care. Attention can and should be drawn to persistent pain through a heightened exposure of the public and professionals to research findings and to suggestions based on these, which concentrate on improving care.

The fact that the current methods for addressing persistent pain in all of its forms need improvement is evident from even a cursory examination of the literature having to do with inadequate pain relief. Additionally, economic 
analyses show the glaring need for a restructuring of the current system in order to relieve the strain on purchasers, providers, and patients alike. Persistent pain is prevalent, it is costly, and it induces potentially severe and debilitating suffering. By addressing the deficits in the present system with regard to identification, information, and care, society can begin to address more effectively the complex set of problems associated with this condition. Care management provides a useful set of principles with which to begin to do this, and implementation of the methods associated with care management would offer comprehensive, individualized alternatives at all levels of treatment that are not typically available today.

To emphasize, the four principles detailed by the APS - identification, appropriate referral, education and assistance to PCPs, and the development of care management plans-could and should be the foundation for an action plan regarding persistent pain. While identification of persistent pain is a challenging issue, the development and widespread use of specialized, validated criteria to identify sufferers will contribute to the successful implementation of this step, as will the placement of an emphasis on patient and provider education. Appropriate referrals, an issue that permeates all points of the healthcare system, from patients to providers to insurance companies, can be facilitated through increased knowledge about persistent pain in general and about the specific networks of care available in different areas. By educating and assisting PCPs to make informed decisions based on the most recent medical evidence and individual patients' needs, early detection of persistent pain, successful implementation of treatment protocols, coordination of services, and cost reduction all become achievable goals. Finally, integrated, individualized care management plans similar to those used for chronic diseases should be developed, systematically implemented, and then periodically evaluated and updated, in order to provide each person with persistent pain the best care possible, as quickly and comprehensively as possible. Now is the time to implement a comprehensive care management strategy to begin to combat the effects of this prevalent, potentially debilitating, and costly condition.

\section{ACKNOWLEDGMENTS}

This review was funded by Purdue Pharma, L.P.

\section{REFERENCES}

1. Gitlin MC. Chronic non-cancer pain: an overview of assessment and contemporary management. $\mathrm{J} \mathrm{La}$ State Med Soc 1999;151:93-98.

2. Pain assessment and treatment in the managed care environment. A position statement from the American Pain Society. Case Manager 2000;11(5):50-53.

3. Fox PL, Parminder R, Alejandro JR. Prevalence and treatment of pain in older adults in nursing homes and other long-term care institutions: a systemic review. Can Med Assoc J 1999;160:329-333.

4. Won A, Lapane K, Givanni G, Roberto B, More V, Lipsitz L. Correlates and management of nonmalignant pain in the nursing home. J Am Geriatr Soc 1999;47: 936-942.

5. AGS Panel on Chronic Pain in Older Persons. The management of chronic pain in older persons. J Am Geriatr Soc 1998;46:635-651.

6. Becker $\mathrm{N}$, Thomsen $\mathrm{AB}$, Korneliuw AO, Sjogren $\mathrm{P}$, Bech P, Eriksen J. Pain epidemiology and health related quality of life in chronic non-malignant pain patients referred to a Danish multi disciplinary pain center. Pain 1997;73:393-400.

7. Wells-Federman CL. Care of the patient with chronic pain: part II. Clin Excell Nurse Pract 2000;4:4-12.

8. American Geriatrics Society. Care management position statement. Available at: http://americangeriatrics. org/products/positionpapers/cmps.shtml. Accessed January 15, 2003.

9. Stratton L. Evaluating the effectiveness of a hospital's pain management program. J Nurs Care Qual 1999; 13(4):8-18.

10. Shimp LA. Safety issues in the pharmacologic management of chronic pain in the elderly. Pharmacotherapy 1998;18:1313-1321.

11. AGS Clinical Practice Guidelines. The management of persistent pain in older persons. Available at: http:// www.americangeria trics.org/education/executive_ summ.shtml. Accessed December 18, 2002.

12. International Association for the Study of Pain. IASP pain terminology. Available at: http://www.iasp-pain. org/terms-p.html\#Pain. Accessed January 15, 2003.

13. Verhaak PJL, Kerssens J, Dekker J, Sorbi JJ, Bensing JM. Prevalence of chronic benign pain disorder among adults: a review of the literature. Pain 1998;77:231239.

14. Marcus DA. Treatment of nonmalignant chronic pain. Am Fam Physician 2000;61:1331-1336.

15. Gureje O, Von Korff M, Simon GE, Gater R. Persistent pain and well-being: a World Health Organization study in primary care. JAMA 1998;280:147-151.

16. Peters L, Simon EP, Folen RA, Umphress V. The 
COPE Program: treatment efficacy and medical utilization outcome of a chronic pain management program at a major military hospital. Milit Med 2000; 165:954-960.

17. Fitzpatrick RJ, Kempler HL. Integrating care for chronic pain patients. HMO Pract 1990;4(3):87-93.

18. Phillips DM. JCAHO pain management standards are unveiled. Joint Commission on Accreditation of Healthcare Organizations. JAMA 2000;284:428-429.

19. Crook J, Rideout E, Browne G. The prevalence of pain complaints in a general population. Pain 1984;18:299 314.

20. Teno JM, Weitzen S, Wetle T, Mor V. Persistent pain in nursing home residents. JAMA 2001;285:2081.

21. Dafault MA, Lessne-Willey C. Using a collaborative research utilization model to develop and test the effects of clinical pathways for pain management. J Nurs Care Qual 1999;13(4):19-33.

22. Katz N. The impact of pain management on quality of life. J Pain Symptom Manage 2002;24(Suppl1):S38-S47.

23. Lerner D, Reed JI, Massarotti E, Wester LM, Burke TA. The Work Limitations Questionnaire's validity and reliability among patients with osteoarthritis. J Clin Epidemiol 2002;55:197-208.

24. Fishman P, Von Korff M, Hecht J. Chronic care costs in managed care. Health Aff 2000;16:239-247.

25. Skevington SM. Investigating the relationship between pain and discomfort and quality of life, using the WHOQOL. Pain 1998;76:395-406.

26. Thomsen AB, Sorensen J, Sjogren P, Eriksen J. Economic evaluation of multidisciplinary pain management in chronic pain patients: a qualitative systematic review. J Pain Symptom Manage 2001;22:688698.

27. Montak SL, Martin J. Treating chronic pain. Am Fam Physician 1997;55:1151-1160.

28. Martin TJ, Eisenach JC. Pharmacology of opioid and nonopioid analgesics in chronic pain states. J Pharmacol Exp Ther 2001;299:811-817.

29. Barkin RL, Lubenow TR, Bruehl S, Husfeldt B, Ivankovich O, Barkin SJ. Management of chronic pain. Part I. Dis Mon 1996;42:389-454.

30. The use of opioids for the treatment of chronic pain. A consensus statement from the American Academy of Pain Medicine and the American Pain Society. Clin J Pain 1997;13:6-8.

31. Joranson DE, Gilson AM. Regulatory barriers to pain management. Semin Oncol Nurs 1998;14:158-163.

32. Paice JA, Toy C, Shott S. Barriers to cancer pain relief: fear of tolerance and addiction. J Pain Symptom Manage 1998;16:1-9.

33. Wells-Federman CL. Care of the patient with chronic pain: Part I. Clin Excell Nurse Pract 1999;3:192-204.

34. McCarberg B, Wolf J. Chronic pain management in a health maintenance organization. Clin J Pain 1999;15: 50-57.

35. Peters J, Large RG, Elkind G. Follow-up results from a randomised controlled trial evaluating in- and outpatient pain management programmes. Pain 1992;50: 41-50.

36. Flor H, Fydrich T, Turk DC. Efficacy of multidisciplinary pain treatment centers: a meta-analytic review. Pain 1992;49:221-230.

37. Gagliese L, Melzack R. Chronic pain in elderly people. Pain 1997;70:3-14.

38. Williams AC, Richardson PH, Nicholas MK, et al. Inpatient vs. outpatient pain management: results of a randomised controlled trial. Pain 1996;66:13-22.

39. Becker N, Sjogren P, Bech P, Olsen AK, Eriksen J. Treatment outcome of chronic non-malignant pain patients managed in a Danish multidisciplinary pain centre compared to general practice: a randomised controlled trial. Pain 2000;84:203-211.

40. Peters JL, Large RG. A randomised control trial evaluating in- and outpatient pain management programmes. Pain 1990;41:283-293.

Address reprint requests to: Christine W. Hartmann, M.S.S. Project Director Office of Health Policy and Clinical Outcomes Thomas Jefferson University 1015 Walnut Street Philadelphia, PA 19107

E-mail: christine.hartmann@jefferson.edu 